\title{
METRETIQUE, ETHIQUE ET POLITIQUE: LE PROTAGORAS ET LE POLITIQUE DE PLATON
}

\author{
Sébastian Bassu \\ PPGFIL - Université de Provence, Aix-Marseille
}

\begin{abstract}
The objective of this paper is precisely to ask ourselves about the ethical and political thought of Plato from what he calls "metretic": can the relationship with the art of measurement provide a relevant context in understanding of his ethics and political philosophy? Indeed, the concept of metretic is closely associated with themes like virtue and the art of politics in the two main dialogues, the Protagoras and the Statesman. We propose to study these two works to understand the function of metretic in the art of acting and the polictical art by Plato.
\end{abstract}

Keywords: Plato, metretic, ethics, politics.

Resumo: O objetivo deste artigo é, precisamente, interrogar-nos sobre a reflexão ética e política de Platão com relação ao que ele chama "metrética": a relação feita com a arte da medida pode constituir uma perspectiva pertinente na compreensão de sua filosofia ética e política? Com efeito, a noção de metrética aparece estreitamente associada aos temas da virtude e da arte política nos dois diálogos principais, o Protágoras e o Político. Propomo-nos a estudar estas duas obras para compreender a função da métrica na arte de agir e a arte política segundo Platão.

Palavras-chave: Platão, metrética, ética, política.

Il est établi que la notion de "mesure" possède une place importante dans la réflexion éthique et politique des Grecs. Cette caractéristique se retrouve en particulier chez Platon qui donne à cette notion une assise philosophique plus précise. Dans la philosophie de Platon, le problème est celui de comprendre la relation entre l'art de la mesure (metrêtikê), l'art d'agir 
et l'art politique. Il n'est pas si commun de considérer l'éthique et la politique dans la philosophie de Platon sous le biais de leurs relations avec l'art de la mesure. Généralement cet aspect de la pensée platonicienne n'est pas mis en lumière, alors qu'il est essentiel. Notre objectif est précisément de nous interroger sur la réflexion éthique et politique du philosophe par rapport à ce qu'il appelle la "métrétique": la relation faite avec l'art de la mesure peut-elle constituer une perspective pertinente dans la compréhension de sa philosophie éthique et politique? Ce questionnement s'enracine directement dans une lecture des textes. En effet, la notion de métrétique apparaît étroitement associée aux thèmes de la vertu et de l'art politique dans deux dialogues principaux, le Protagoras et le Politique. Huit acceptions du terme metrêtikê se trouvent dans le Protagoras concentrées sur quelques pages ${ }^{1}$. Dans le contexte même du passage, il s'agit de déterminer la fonction de la métrétique dans l'art d'agir (to eu prattein) et l'art de mener sa vie: l'art de mesurer est clairement présenté comme essentiel dans le fait de bien agir et de préserver "le salut de note vie" (sôtêria tou biou) ${ }^{2}$. Le texte précise bien qu'il s'agit de l'action (praxis) en général et de l'agir (prattein). Or, dans le Protagoras, ce questionnement est lié à l'enquête sur la notion de vertu (aretê) et en tout premier lieu, à la vertu politique (politikê). De même, dans le Politique, Platon associe de nouveau la métrétique à l'art politique, aux actes (praxeis) et aux arts (technai) en général. L'art de la mesure est présenté comme une condition de possibilité de la réussite de nos actes ${ }^{3}$. Nous nous proposons d'étudier ces deux extraits pour comprendre la fonction de la métrétique dans l'art d'agir et l'art politique selon Platon. Le principe qui fonde la position platonicienne est de faire de la science (epistêmê) le fondement de la vertu et des arts. C'est pourquoi nous nous demanderons, enfin, si la dialectique possède une fonction dans la constitution de ces arts, et corrélativement les liens qu'elle entretient avec les notions de "mesure" et de "métrétique" en convoquant un dernier dialogue qui est celui du Philèbe. Dans cette perspective, nous commencerons par le problème de la relation entre métrétique, vertu et science dans le Protagoras.

1356 d 4; 356 d 8; 356 e 3; 356 e 4; 357 a 1; 357 b 2; 357 b 4; 357 d 7.

$2356 \mathrm{~d} 1-4$.

${ }^{3}$ Le passage sur la métrétique s'étend de $283 \mathrm{~b}-286 \mathrm{~d}$. 


\section{La métrétique dans le Protagoras de Platon.}

Cette étude sur le Protagoras s'effectuera en trois moments: premièrement, l'étude de l'extrait en lui-même; deuxièmement, l'insertion de notre interprétation dans la compréhension générale du dialogue; enfin, la mise en relation de ce passage avec d'autres textes pouvant (ou non) l'éclaircir. Dans le passage désigné, Socrate ne se limite pas à évoquer la possibilité de la métrétique, il fait plutôt de cet art ou science une condition de possibilité de la vertu: "Si donc la vertu de l'action (to eu prattein) dépendait pour nous de notre attention à choisir dans nos actes les plus grandes dimensions et à éviter les plus petites, qu'est-ce qui nous apparaîtrait comme la condition de notre salut (sôtêria tou biou)? Serait-ce l'art de mesurer? [...] D'où viendrait notre salut? N'est-ce pas d'un savoir exact (epistêmê)? Et encore d'une certaine science de la mesure (metrêtikê), puisque celle-ci est la science de l'excès et du défaut (huperbolês te kai endeias estin hê technê) $)^{4}$ ?" L'essentiel de la position socratique se trouve dans l'exigence de fonder l'art d'agir sur la science. La métrétique se présente alors comme une telle science à propos de tout ce qui relève de l'excès et du défaut. Avant d'analyser ce point, rappelons que la métrétique est introduite après une surprenante "thèse" de Socrate selon laquelle ce qui est "bon" se confond avec ce qui est agréable. Socrate semble endosser une thèse hédoniste en proposant une assimilation entre ce qui est bon (agatha) et ce qui est agréable (hêdea), ${ }^{5}$ qui devient une identification entre la vie agréable et ce qui est bon (hêdeôs zên agathon) ${ }^{6}$. Inversement, la vie non agréable est mauvaise (to d'andrôs kakon). Socrate fait alors remarquer que le plaisir (hêdonê) et la douleur (to lupeithai; lupê) sont de l'ordre du plus et du moins, de l'excès et du défaut. En ce sens, il est possible de distinguer les plaisirs et les peines en évaluant leurs quantités ou leurs qualités et en les comparant. Ces deux principes, susceptibles d'excès et de défaut, peuvent donc faire partie des objets sur lesquels la métrétique agit. C'est, en effet, à ce stade du raisonnement que le philosophe compare celui qui choisit entre un plaisir et

${ }^{4}$ Protagoras, $356 \mathrm{~d} 1-357$ a 2.

$5351 \mathrm{c}$.

$6351 \mathrm{e}$. 
une peine et celui qui "sait peser correctement"7. L'art de la mesure est ainsi introduit apparemment comme technique d'évaluation de présence ou d'absence de valeur des plaisirs et des peines ${ }^{8}$. A ce stade, la présentation simple d'une science pratique évaluant les plaisirs et les peines devient problématique, puisque la position hédoniste de Socrate suscite elle-même des interrogations ${ }^{9}$. Or, ce problème a des conséquences sur le sens à donner à la métrétique. Ramenée au problème du rôle de la métrétique dans la vie pratique, la question est de savoir si Socrate se présente vraiment comme hédoniste et donne en ce sens à la métrétique la tâche d'opérer un calcul des plaisirs ou bien si le développement de Socrate relève d'une pure stratégie réfutative ${ }^{10}$. Or, les deux hypothèses n'apparaissent pas convaincantes. En effet, la première est peu probable en comparaison à l'ensemble de l'œuvre de Platon qui ne fait pas de Socrate un hédoniste; la seconde fait de la métrétique une idée subordonnée à une critique ad hominem ce qui ne convient pas à l'importance donnée à l'idée de mesure dans de nombreux dialogues de maturité. Quelle position faut-il adopter ${ }^{11}$ ?

Une des caractéristiques de la méthode socratique est d'examiner différentes thèses en présence, admises soit par l'interlocuteur, soit par le plus grand nombre, soit encore suscitées par Socrate lui-même. Par conséquent, certaines peuvent être prêtées à Socrate et non d'autres. Encore faut-il nuancer le fait d'attribuer à Socrate telle ou telle thèse puisque Socrate n'est jamais censé exprimer sa propre pensée dans les dialogues socratiques. Pourtant,

\footnotetext{
7356 a $8-356$ b 1.

8356 a 1 « Mais quelle est la valeur d'un plaisir par rapport à une peine, si ce n'est son excès ou son défaut par rapport à elles » (kai tis allê anaxia hêdonêi pros lupen estin, all' ê huperbolê allêlôn kai elleipsis) ».

${ }^{9}$ Certains auteurs acceptent la théorie hédoniste présentée dans le Protagoras comme appartenant effectivement à une position socratique ou socratico-platonicienne. Mentionnons G. Grote, Plato and the Other Companions of Socrates, Londres, 1988, pp. 87 - 89; R. Hackforth, « Hedonism in Plato's Protagoras », Classical Quaterly, 22, 1928, pp. 39 - 42;; J.C.B Gosling et C.C.W. Taylor, The Greeks on Pleasure, Oxford, 1982, pp. $65-68$.

10 Une étude représentative de cette position est celle de D. J. Zeyl, "Socrates and Hedonism: Protagoras 351 b-358d », Phronesis, 25, 1980, pp. $250-269$.

${ }^{11}$ La position de Juila Annas nous semble caractéristique de la difficulté à trancher la place de la théorie hédoniste du Protagoras: « La théorie avancée dans le Protagoras n'y apparaît pas comme acceptée par Socrate: il l'examine dans un esprit de recherche. Elle n'est cependant pas attribuée non plus à Protagoras, et elle n'est pas définitivement rejetée ». («Platon» dans Jacques Brunschwig et G. E. R. Lloyd (éds.), Le Savoir grec, Paris, 1996, pp. 746 - 747). Nous nous situons dans cette interprétation qui ne fait pas de l'hédonisme une position de Socrate mais un moyen discursif en vue de parvenir à la question de la science comme fondement de l'action et du choix. La position de Gregory Vlastos semble ne pas vouloir non plus trancher de manière résolue la difficulté: Plato, Protagoras, Indianapolis, 1956.
} 
nous pouvons nous demander quelles sont les thèses ou les critiques principales qui représenteraient le mieux la pensée du personnage Socrate et de son auteur Platon. Conformément à la structure des dialogues socratiques, la question initiale, savoir si la vertu peut être enseignée, ne fait qu'introduire une question préalable pour Socrate, savoir ce qu'est la chose dont nous parlons. Le problème de la définition de la vertu est donc le sujet essentiel du dialogue. Celui-ci se décline, d'après notre lecture, en plusieurs axes. La question principale est de savoir si la vertu relève de la science ou non? Le problème de savoir si les différentes vertus, sagesse, prudence, justice, courage et sainteté (sophia kai sôphrôsinê kai andreia kai dikaiosunê kai horiotês) sont une seule et même chose, ou si elles sont distinctes ${ }^{12}$ est, nous semble-til, subordonné à la première interrogation. En effet, la réponse à la seconde question mène à la première: si les vertus sont unes, c'est parce qu'elles relèvent toutes de la science. Et c'est en tant qu'elles relèvent toutes de la science, même le courage, qu'elles s'unissent. Or, sur quoi porte particulièrement la critique de Socrate à l'égard de Protagoras? Elle porte sur l'essence même de la vertu. Pour Protagoras, la vertu n'est pas une science, et la contradiction apparente à laquelle aboutit l'examen de Socrate est de soutenir à la fois que la vertu s'enseigne, alors qu'elle n'est pas une science ${ }^{13}$.

Notre hypothèse consiste donc à considérer que l'intervention de la métrétique a pour seul but de montrer que la vertu relève de la science, même dans le cas où nous adoptons une position hédoniste. En effet, cette position est souvent liée à l'idée que le plaisir est le principe de détermination de notre vie, niant à la science la valeur de motif déterminant. Le but de ce passage est de montrer que la science a un véritable pouvoir sur le choix des hommes, et un pouvoir plus grand que le plaisir. Socrate en endossant l'hédonisme le plus simple, montre que même dans de telles conditions la science possède toujours une fonction directrice. L'erreur dans la poursuite des plaisirs et des

12349 b $1-5$. «Ce qu'on appelle savoir, sagesse, courage, justice et sainteté, sont-ce cinq noms différents pour une seule et même chose, ou chacun de ces noms correspond-il à une réalité distincte (epi eni pragmati estin), à un objet ayant son caractère propre (tis idios ousia), et tel que l'un ne puisse être identifié avec l'autre. »

${ }_{13} 361 \mathrm{a}$ - b. « II me semble que notre discours même, en arrivant à sa conclusion, devient comme notre accusateur et se moque de nous [...]: " vous êtes de plaisants personnages, Socrate et Protagoras: toi, Socrate, qui niais d'abord que la vertu pût s'enseigner, voici que tu mets tous tes efforts à te contredire en démontrant que tout est science, la justice, la tempérance, le courage, ce qui est le plus sûr moyen de montrer qu'on peut enseigner la vertu. [...] D'autre part, Protagoras, qui avait d'abord mis en fait qu'elle se pouvait enseigner, semble maintenant s'appliquer à se contredire, voyant en elle tout plutôt qu'une science, ce qui lui ôterait toute possibilité d'être enseignée." » 
peines ne vient pas du fait que nous avons été contraints par le plaisir à choisir telle ou telle issue, mais de l'ignorance. Notre hypothèse de lecture s'inscrit dans cette description: la métrétique intervient pour montrer que toute vertu et toute action réussie reposent sur la science. Voilà le point essentiel de la démarche socratique, et l'hédonisme n'est qu'une opinion endossée en vue d'une telle démonstration. Ce qui montre implicitement que le plaisir et le bien ne sont pas totalement identiques, c'est justement la nécessité de la science métrétique comme condition de possibilité de la vie heureuse. Autrement dit, le plaisir ne se suffit pas lui-même puisqu'il faut lui ajouter la science et la vertu. Platon n'a jamais refusé une quelconque valeur au plaisir et a souvent reconnu le fait que la vie humaine était concernée par le principe du plaisir et de la peine. Dans le Philèbe, il fait une large place à l'examen de la vie mixte faite de plaisir et de sagesse. Le Protagoras est certainement une annonce d'une telle recherche. De même, dans la République Platon fait référence à la nécessité d'éduquer les hommes en prenant en compte les plaisirs et les peines. Le plaisir ne peut être tout au plus qu'un aspect de la vie heureuse menée par les hommes; abandonné à luimême le plaisir est incapable de faire la vie heureuse. Car Socrate cherche à montrer que la science est supérieure au plaisir ${ }^{14}$ et que l'homme qui s'adonne à une vie de plaisirs abandonnée à elle-même ne sera jamais homme de bien. Socrate s'efforce de critiquer l'opinion commune selon laquelle "il n'y a en elle aucune force, aucune puissance de direction et de commandement (dokei de tois pollois peri epistêmês touiouton ti, ouk ischuron oud' hêgemonikon oud' archikon einai)". C'est à la métrétique que revient la tâche d'illustrer la puissance directrice de la science dans l'art d'agir, c'est-à-dire en un mot dans la vertu. Le dialogue n'est pas le lieu d'une investigation sur ce qu'est le bien, mais plutôt sur ce qu'est la vertu et la vie réussie ${ }^{15}$ : dans cette perspective, ce qu'il fallait montrer c'est que la vertu repose sur la science et que la science est supérieuer au plaisir. Nous pouvons donc penser que la métrétique possède une double fonction: elle fait de la vertu une science, et elle inscrit cette vertu dans la vie pratique et politique ${ }^{16}$. Que la métrétique apparaisse

\footnotetext{
$14352 \mathrm{~b}-\mathrm{d}$.

15360 e 7 - 361 a 3. « Si je te pose toutes ces questions, c'est uniquement pour voir ce qu'il en est de la vertu, et en quoi consiste cette chose qu'on appelle la vertu. Je suis sûr que, cette question une fois résolue, il sera facile d'élucider celle qui a provoqué de notre part à tous deux de si longs discours, moi, soutenant que la vertu ne peut s'enseigner, et toi, qu'elle le peut ».

${ }^{16} \mathrm{Si}$, par exemple, nous nous référons au Gorgias, nous constatons que la critique adressée à Calliclès qui défend un hédonisme démesuré consiste à montrer que la vertu réside dans l'ordre et la mesure. Ces
} 
comme un calcul des plaisirs et des peines n'est qu'un aspect de l'usage fait par Platon de la notion de métrétique. Cependant, là où on l'attend le moins, la science et la rationalité exercent un commandement ${ }^{17}$.

Considérons maintenant l'extrait qui fait référence à la métrétique et demandons-nous s'il confirme notre hypothèse. Ensuite, revenons à notre questionnement principal, savoir quelle est la fonction de la métrétique dans l'art d'agir. Le problème est clairement posé: comment l'individu choisit-il de réaliser un plaisir quand celui-ci suppose aussi une peine? Il s'agit donc de comprendre comment l'homme détermine la valeur du plaisir ou de la peine, ainsi que sa quantité. "Quelle valeur donner à un plaisir relativement à une peine, si ce n'est le fait que l'un a sur l'autre de l'excès ou du défaut? (anaxion ontôn nikar. Kai tis allê anaxia hêdonêi pros lupên estin, all' ê huperbolê allêlon kai elleipsis)" 18 . Il faut alors réaliser une évaluation comparable à la pesée dans la mesure où le plaisir et la peine sont des réalités changeantes (ta gignomena) qui sont concernées par le plus et le moins, le plus grand et le plus petit, le plus intense et le moins intense: "comme un homme qui sait peser correctement, pose d'un côté les qualités agréables, de l'autre les qualités pénibles, place aussi dans la balance l'immédiat et le différé, et dis-moi alors lequel des deux l'emporte?"19. Deux dimensions caractérisent l'évaluation des plaisirs et des peines: l'une qui est quantitative ou qualitative, l'autre qui est temporel puisque le choix porte aussi sur le fait de mettre en balance un plaisir immédiat avec une peine à venir. La balance est une métaphore du jugement. Par là, Socrate montre que la praxis suppose toujours au préalable un jugement sur ce que nous allons faire. Agir suppose une évaluation de la qualité de notre action, et, dans le cas présent, une évaluation du plaisir que nous recherchons ou de la peine que nous fuyons. Cette idée est un leitmotiv

deux dialogues ne sont donc pas si contradictoires, puisque les deux opposent à la vie de plaisirs, le mode de vie philosophique dont le maître mot est la juste mesure.

${ }^{17}$ Dans cette perspective, nous pouvons faire référence au commentaire de Martha $\mathrm{C}$. Nussbaum sur le Protagoras dans son ouvrage intitulé The Fragility of Goodness. Luck and Ethics in Greek Tragedy and Philosophy, London/New York: Cambridge University Press, 1986. L'auteur développe l'idée qu'une véritable "science pratique » (a science of practical reasoning) est constituée dans le passage du Protagoras que nous allons étudié. Voir aussi T. Irwin qui défend aussi la position d'une «science pratique » dans le Protagoras: T. Irwin, Plato's Moral Theory, Oxford, 1977. Nous sommes d'accord avec Irwin pour dire que le principe soutenu par Socrate dans le dialogue est l'identification entre vertu et science. Cependant, nous ne pensons pas que la science pratique désignée comme métrétique ait un sens instrumental. II ne s'agit pas de déterminer les moyens pour parvenir à l'efficacité de l'action, il est préférable de parler de détermination de ce qui est bon ou non de faire.

18356 a $1-3$.

19356 a $8-356$ b 2. 
de la critique de Platon à l'égard de l'hédonisme. Ainsi, dans le Philèbe, Socrate s'interroge sur la valeur d'une vie faite uniquement de plaisirs sans pensée comme celle du "poumon marin"20? Serait-elle vraiment digne d'être vécue? De même dans le Théétète, Socrate moque la thèse sophistique de l'homme-mesure en demandant si le cynocéphale ne pourrait pas être aussi mesure puisqu'il éprouve des sensations (aisthêsis) ${ }^{21}$. Socrate rappelle une nouvelle fois la nécessité pour la vie humaine de ne pas se limiter aux sensations, aux plaisirs et aux peines, mais de toujours faire intervenir le jugement, le logos, en un mot la science (epistêmê). La métrétique se présente donc comme une capacité à juger droitement. Par conséquent, bien agir (to eu prattein) exige la capacité à mesurer la valeur de nos actes, du plaisir et de la peine. Cette capacité ne peut se limiter à un jugement portant sur les apparences ou les phénomènes, mais suppose un véritable art, la métrétique. La métrétique se définit donc d'emblée comme un art qui ne porte pas sur les apparences ou les phénomènes. "Si le fait de bien agir consistait pour nous en ce que nous réalisons et suivons les grandes parties, et en ce que nous fuyons et ne réalisons pas les plus petites, quel salut pour notre vie nous apparaitrait alors? Serait- ce l'art de mesurer ou la puissance des apparences?”22. Il est possible de voir dans ce passage une adresse faite à la doctrine de Protagoras selon laquelle la connaissance est sensation et que, par conséquent, la connaissance porte sur ce qui nous apparaît. Platon, par l'intermédiaire de la critique socratique, montre les insuffisances de la position du sophiste concernant la capacité à bien agir, c'est-à-dire la vertu. Il entend souligner l'impossibilité de fonder une vertu sur la connaissance des phénomènes, car ceux-ci sans cesse changeants et relatifs dans leur nature même ne peuvent être pris pour critères dans l'évaluation du bien ni même du vrai. "N'est-il pas vrai que la puissance des apparences nous égarerait, nous ferait prendre sans cesse les grandeurs les unes pour les autres et serait pour nous une cause de perpétuels repentirs dans nos actes et dans nos appréciations du grand et du petit?" 23 . La métrétique est dès lors considérée comme une connaissance exacte de la qualité des choses qui déjoue les illusions causées par les phénomènes. A ce point du dialogue, nous pourrions tout à fait imaginer un renversement de Socrate à l'adresse de son prétendu

\footnotetext{
20 Philèbe, $21 \mathrm{c}$.

$21161 \mathrm{c}$.

$22356 \mathrm{~d} 1-5$.

${ }^{23} 356 \mathrm{~d} 5-8$.
} 
"hédonisme" en montrant que le plaisir n'est pas en soi le Bien puisqu'il doit toujours être subordonné à la connaissance du Bien. S'élever à cette connaissance aurait pour conséquence le fait de nuancer l'hédonisme. La constitution de ce renversement suppose la possibilité de s'élever au-delà des phénomènes et des apparences. Cette possibilité indique l'existence d'une science des Idées, c'est-à-dire du Grand et du Petit en soi. Par conséquent, l'argument de Platon ne se limite pas à une critique ad hominem mais indique la position du philosophe. La finalité même de l'argumentation le prouve puisque ce détour par l'art de la mesure était guidé par l'intention de montrer que bien agir, c'est-à-dire la vertu, relève de la connaissance, et que mal agir, au contraire, trouvait son origine dans l'ignorance, position caractéristique de Socrate et de Platon. "Vous avez reconnu que c'était le défaut de la science qui faisait faire un mauvais choix entre les plaisirs et les peines [...]. Non seulement il y avait là défaut de science, mais en outre d'une science que vous avez reconnue être celle de la mesure" 24 . Le to eu prattein relève donc de la technê et de l'epistêmê: "puisque c'est la métrétique, c'est nécessairement un art et une science" 25 . Que permet en effet l'art de la mesure par opposition aux phénomènes? D'abord, elle permet de rendre sans effets les impressions laissées par les phénomènes sur l'âme, impressions équivoques et ambiguës. Cette ambiguïté des phénomènes, savoir l'impossibilité de définir la valeur des qualités de manière exacte, a pour effet de jeter l'âme dans le trouble et l'empêcher d'opérer un choix de manière assurée. "L'art de la mesure réduirait à l'impuissance cette apparence sensible (to phantasma)"26. Si l'art de la mesure possède une telle capacité, c'est en raison de son fondement épistémique: l'art de la mesure est une détermination de la vérité. "Par la connaissance de la vérité, l'art de la mesure assurerait à nos âmes une stabilité tranquille dans la possession du vrai et ferait le salut de notre vie" 27 . Socrate insiste de manière définitive sur le rapport entre art de la mesure et science, c'est-à-dire entre mesure et vérité28. Pour illustrer cette exactitude de l'art de la mesure, Socrate prend l'exemple de l'arithmétique. L'arithmétique est en effet présentée comme une

\footnotetext{
$24357 \mathrm{~d} 3-8$.

25357 b $3-4$.

${ }^{26} 356 \mathrm{~d} 8-9$.

27356 e $1-3$.

${ }^{28}$ Cf. République, $486 \mathrm{~d}$ - e. "La vérité (alêtheian) est [dite] parente de la mesure (sungenê einai ê emmetriai)».
} 
métrétique dont l'objet est le pair et l'impair. C'est à cette connaissance que nous aurions recours en théorie, concernant le jugement sur les nombres euxmêmes, mais aussi en pratique concernant par exemple l'estimation des distances ${ }^{29}$. Il n'y a pas de séparation entre la théorie et la pratique dans la mesure où l'acte réussi repose sur une droite détermination de l'essence des choses. Dans ce contexte, d'un point de vue éthique, le problème apparent de l'akrasia trouve aussi sa résolution. A proprement parler, celle-ci n'existe pas, mais l'acte manqué est plutôt de l'ordre de l'ignorance, thèse clairement prêtée à Socrate par Aristote. C'est, semble-t-il, la conséquence de l'idée qu'il existe une science pratique qui est à l'origine de nos choix. Si la science est commandement, l'homme ne peut être acratique au sens où il renoncerait à suivre le juste choix emporté par le désir ${ }^{30}$. Le prétendu hédonisme de Socrate participe aussi d'une critique de l'idée selon laquelle l'homme connaissant le bien puisse, pourtant, commettre un acte mauvais parce qu'il serait entraîné par un désir immédiat. Tout cela revient à rappeler la primauté de la connaissance sur le plaisir dans la direction de l'âme. Une autre caractéristique de la métrétique tient à la nature de son objet: $\mathrm{ce}^{31}$ qui accepte l'excès et le défaut ${ }^{32}$. Dans ce passage, le plaisir et la peine font partie de cet ensemble d'objets. Cette caractéristique de la métrétique est développée

\footnotetext{
${ }^{29}$ «Et si notre salut dépendait du choix entre le pair et l'impair, si nous devions tenir compte du plus ou du moins, soit dans la comparaison d'un nombre avec lui-même soit dans les rapports entre plusieurs nombres, et en outre de la distance où nous serions placés, d'où nous viendrait notre salut? N'est-ce pas d'une science? Et encore d'une certaine science de la mesure, puisque celle-ci est la science de l'excès et du manque? Et puisqu'il s'agit du pair et de l'impair, ne serait-ce pas de l'arithmétique? » 356 e 5 - 357 a 3.

${ }_{30}$ Protagoras, 355 a 5 - b 3. « Je vous déclare que, s'il en est ainsi, votre langage est absurde lorsque vous dites que souvent un homme, connaissant qu'une chose est mauvaise, l'accomplit cependant, sans y être forcé, parce qu'il est entraîné et égaré par le plaisir. » Cette position est clairement défendue par Donald J. Zeyl, "Socrates and Hedonism», 1980 qui argumente en faveur de la position « antihedonist », p. 250.

${ }^{31}$ Cette question devra être posée dans la suite de l'étude: la métrétique s'applique-t-elle à la Réalité ou au devenir. Une réponse possible est de considérer que l'art de la mesure est une connaissance des essences, mais qu'elle est un art appliqué au devenir. II s'agit d'appliquer la connaissance des essences aux actions et arts, activités se réalisant dans le devenir.

32 Philèbe, $24 \mathrm{a}-25 \mathrm{~b}$; Politique, $283 \mathrm{~b}-285 \mathrm{c}$. «A la longueur, à la brièveté, à tout ce qui est excès ou défaut; car c'est à tout cela, je crois, que s'applique l'art de la mesure », 283 c $11-\mathrm{d} 2$ 2. La distinction entre quantitatif et qualitatif n'est pas toujours significative. En effet, la mesure insiste sur un aspect quantitatif, mais, d'un autre côté, l'évaluation de la métrétique porte tout aussi bien sur ce que nous désignerions aujourd'hui comme des qualités. Le cas du plaisir est à ce sujet pertinent: il peut être mesuré à la fois quantitativement et qualitativement. A ce sujet, il ne faut pas d'emblée présumer de la primauté de la quantification sur l'aspect qualitatif. L'analogie avec la pesée ne restreint pas l'art de la mesure à une quantification.
} 
dans deux autres dialogues, le Politique et le Philèbe. Comme nous le verrons prochainement, le Politique introduit l'art de la mesure à partir d'une considération sur l'excès et le défaut concernant les discours ${ }^{33}$. Mais c'est dans le Philèbe que ce rapport est réalisé avec plus de précision. En effet, la mesure, metron, est définie comme ce qui permet de réaliser dans tout ce qui est de l'ordre du plus et du moins l'achèvement et la quantité définie ${ }^{34}$. La métrétique est la technique permettant d'appliquer la mesure aux qualités en excès et défaut, et produire ainsi la "juste mesure (metrion)". Ces qualités sont par exemple, "le plus chaud" et le "plus froid", le "fortement" et le "doucement", le "plus grand" et le "plus petit", et ainsi de suite. L'introduction de la mesure permet de transformer ce qui est illimité (apeiron) en limité (peiras), de faire passer les qualités extrêmes et indéterminées entre les contraires en qualités mesurées et déterminées. La mesure permet de produire le telos dans ce qui est atêlês, inachevé. Or, dans ce passage du Philèbe, il est clairement dit que "le plaisir est illimité (apeiros) par lui-même (autê) et rentre dans un genre (genous) qui n'a et n'aura jamais, en soi et par soi (en autôi aph' eautou), ni commencement, ni milieu, ni fin

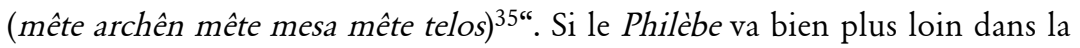
détermination de la fonction du metron, il s'accorde avec le Protagoras sur l'idée que la mesure est ce qui permet de juger la valeur d'une qualité en lui ôtant son indétermination. Il nous faut donc reconnaître que dans le Protagoras aussi, l'ensemble de la praxis est finalement soumise à la nécessité d'évaluer les qualités et les quantités selon une mesure précise. L'action est toujours une évaluation de la qualité de l'acte envisagé ou de l'objet recherché ou fui. Cet acte d'évaluation repose d'abord sur une considération des qualités contraires, et ensuite sur la détermination exacte de la qualité de l'objet.

Finalement, la métrétique du Protagoras est véritablement présentée comme une science "pratique" 36 , c'est-à-dire une science qui permet à

\footnotetext{
${ }^{33}$ Politique, 283 c 3 sqq.

34 Philèbe, 24 c. " Partout où ils [le plus et le moins] sont, ils empêchent la réalisation d'une quantité définie, et toujours, au contraire, introduisant dans toute action l'opposition du plus violent au plus paisible et inversement, ils engendrent le plus et le moins et font disparaître la quantité définie. Si au lieu de faire disparaitre la quantité définie, ils la laissaient s'installer, elle et la mesure, là où résident le plus et le moins, ce serait à ceux-ci de fuir la place où ils étaient. »

${ }_{35}$ Philèbe, 31 a 9 - 11. Une difficulté se pose concernant le statut du plaisir dans le Philèbe puisque plus loin dans le dialogue le plaisir semble ne plus faire partie de l'illimité, mais ce n'est pas notre sujet.

${ }^{36}$ Voir à ce sujet l'article suivant: Henry S. Richardson, « Measurement, Pleasure, and Practical Science in Plato's Protagoras », Journal of the History of Philosophy, 28, n 1, 1990, pp. $7-32$.
} 
l'homme de bien agir (to eu prattein) et de préserver sa vie (sôteria tou biou). Cette application à l'action concrète en fait un art. Par la répétition de ces termes, Platon veut faire de la mesure un fondement du mode de vie philosophique dans son aspect pratique. L'art de la mesure est une science pratique dans la mesure où sa finalité est de déterminer le choix et l'action humaine. Cette détermination ne porte pas, selon nous, sur les moyens à utiliser, mais plutôt sur la connaissance de ce qu'est le bien. Il faut entendre le bien de manière objective, c'est-à-dire un bien qui fait l'objet d'une connaissance qui dépasse les simples apparences et les opinions de chacun. Platon indique seulement la possibilité de cette objectivité sans en développer le sens ni aller plus loin dans la séparation du bien à l'égard du sensible. Ensuite, l'art de la mesure participe au mode de vie philosophique, et devient même comme une science du philosophe. En effet, le thème de l'art de la mesure apparaît pour conclure une série de réflexions qui présente un ensemble de vertus et d'arts participant à la sagesse (sophia) de l'homme bon (agathos). La mesure vient conclure le cheminement menant à la description du sage. Autrement dit, c'est le mode de vie du philosophe que Platon présente sous la figure et le discours de Socrate. Ce n'est certainement pas un hasard si le thème des sept sages intervient de manière centrale dans le dialogue avec le rappel du style laconique et des plus célèbres apophtegmes «Gnôthi tauton» et "mêden 'agan» ${ }^{37}$. Le thème de la mesure est lié à cette tradition des sept sages et de la sagesse apollinienne. C'est "l'amour de la sagesse” (philosophia) qui ouvre le discours sur la sagesse lacédomienne ${ }^{38}$. Platon tire profit de la tradition pour la placer du côté de la philosophie, même si le vocabulaire est ambigu, puisqu'il utilise à multiples reprises le terme sophistês, qu'il faut certainement entendre dans son sens général. Il défend le "style" philosophique dont l'aspect rhétorique est la forme dialogique sous forme de questions - réponses caractérisées par leur brièveté: la brachulogie ${ }^{39}$. Socrate s'inscrit bel et bien dans une philosophie de la juste mesure et du savoir comme fondement de la vertu. Il oppose la tradition philosophique à la "tradition sophistique" revendiquée par Protagoras ${ }^{40}$.

37343 b 3.

38342 a 7.

39342 e 2: « logou brakhu »; 343 b 5 « brakhulogia ».

40316 d $3-317$ c 5 . Ce fameux passage commence par les vers suivants puis nomment tous les " sophistes dissimulés ». "J'affirme, quant à moi, que l'art de la sophistique est ancien, mais que ceux des anciens qui pratiquaient cet art avaient coutume de le déguiser et de le dissimuler sous des masques divers ». Le discours de Socrate sur les sept sages est une réponse à cette revendication. La philosophie 
Même son interprétation des vers de Simonide en vient à une telle conclusion annonçant la suite de la discussion: «Mais en quoi consiste le succès (agathê praxis) en matière d'écriture, c'est évidemment qu'il a appris (mathêsis) les lettres" ${ }^{\prime 1}$. Il en de même pour le médecin, le pilote, etc. A l'inverse, «le seul mal véritable (kakê praxis) est la perte du savoir (epistêmês sterêthênar)» ${ }^{42}$. Certes faut-il reconnaître qu'il s'agit de techniques et non de vertus. Cependant, il est très vraisemblable que Socrate utilise ces paradigmes pour illustrer ce qu'il en est de la vertu. La différence essentielle tient en ce que les vertus demandent une science beaucoup plus belle. Dans ce mode de vie philosophique, la juste mesure est aussi présentée comme un juste milieu. Ainsi en $346 \mathrm{~d}$, Socrate interprète les vers de Simonide selon le schème du juste milieu. D'après cette interprétation, le sens du mot de Simonide est le suivant: "il me suffit qu'un homme ne soit par trop mauvais ou par trop (mêden 'agan) incapable"43. Socrate interprète ce mot selon l'idée de juste mesure et de juste milieu. Ce n'est pas la perfection qui est recherchée, mais "le juste milieu (ta mesa) suffit pour qu'on soit à l'abri du blâme" 44 ou "il me suffit d'une qualité moyenne (mesos) qui ne soit pas tout à fait mauvaise (mêden kakon) ${ }^{45}$.

peut elle-aussi faire valoir une tradition non dissimulée, mais reconnue « universellement » pour sa sagesse.

41345 a.

42345 b 5 .

43346 c $2-3$.

$44346 \mathrm{~d} 3$.

$45346 \mathrm{~d} 7-8$. II va sans dire que Platon reprend à son compte une tradition déjà amorcé par Hésiode et développé ensuite par les sept sages et la poésie élégiaque comme celle de Théognis. D'un autre côté, Platon annonce la conceptualisation faite par Aristote du terme meson. Certes, Platon ne constitue pas celle-ci de manière centrale contrairement à Aristote. Nous allons la fonction de cette notion dans les arts et les sciences dont la dialectique, telle qu'elle est présentée dans le Politique de manière très sommaire et dans le Philèbe avec un peu plus de consistance. Notons, cependant, que la notion de metron revêt en partie quelques idées comparables à la notion de meson chez Aristote. En effet, la mesure permet d'en finir avec l'excès et le défaut, de leur donner une détermination et un achèvement. En ce sens, ce qui est mesuré (metrion) par la mesure (metron) est ce qui est en soi réussi et pour ainsi dire parfait, à la fois beau et bon. Or, le meson aristotélicien désigne bien cette propriété de l'action qui lui permet de dépasser les deux écueils, les deux échecs ou les deux vices de l'excès et du défaut en produisant une caractéristique propre à l'acte, achevé et réussi. La métrétique est clairement identifié à une mesure de l'excès et du défaut qui par l'application d'une telle technique disparaissent pour laisser place au « convenable, approprié, opportun et milieu ». D'une certaine manière, le phronimos aristotélicien serait en termes platoniciens celui qui détient la métrétique, à ceci près que la métrétique platonicienne repose, à notre avis, sur la connaissance dialectique, alors que le philosophe de Stagire insiste sur une autonomie du pratique à l'égard du théorique (ou de l'épistémique). S'agit-il d'une épistèmè praktikè chez Aristote? Les objets sont les mêmes, mais la manière de concevoir l'action, la vertu, la technique et la fonction de la science se différencient. 
Le Protagoras, comme nous le verrons au sujet du Politique, semble contredire la doctrine professée dans la République. C'est cette difficulté qui rend le travail interprétatif délicat. Dans la République, les mesures appliquées à des réalités sensibles sont dévalorisées, comment se fait-il alors que dans d'autres dialogues, Platon semble présenter la possibilité de sciences pratiques et politiques? Ce n'est pas seulement une question d' "évolution" de la pensée du philosophe, c'est lier plus fondamentalement à l'hésitation de Platon concernant l'opposition entre sensible et intelligible. Or, force est de constater que Platon donne à ce premier ordre une importance et une réalité beaucoup plus grandes que nous le pensons. En revanche, il faut bien comprendre que les activités pratiques qui se déroulent dans la vie humaine doivent toujours leur excellence à la connaissance intelligible. Cette primauté de la connaissance sur l'activité pratique est essentielle. Elle se trouve une nouvelle fois formulée dans le Politique, dialogue dans lequel métrétique, science et politique sont étroitement liées.

\section{La métrétique en Politique (283 b - 286 b).}

Dans le dialogue du Politique, Platon distingue deux sortes de métrétique. Les commentateurs s'accordent généralement à voir dans la première une métrétique dite "mathématique", et dans la seconde une métrétique "pratique" 46 . Si la seconde métrétique est développée par Platon d'un point de vue général, il ne faut pas perdre de vue qu'elle est censée participer à la définition du "politique". Ne faut-il pas prendre au sérieux l'assimilation entre politique et métrétique parce que Platon déclare que l'ensemble du discours est un exercice à la dialectique ${ }^{47}$. La définition de l'art politique ne serait pas l'objet principal du discours. Il est, au contraire, plus juste de dire que Platon ne propose pas dans le dialogue une définition

\footnotetext{
${ }^{46}$ Cette distinction est opérée comme telle par les principaux commentateurs du passage dont nous donnons une liste non exhaustive: Paul Kucharski, "La conception de l'art de la mesure dans le Politique », Bulletin de l'Association Guillaume Budé, $n^{\circ}$ 3, 1960, pp. 459 - 480; Sylvain Delcomminette, "La juste mesure, Etude sur les rapports entre le Politique et le Philèbe », Les Etudes Philosophiques, Février 2005, n², pp. 347 - 369; Alonso Tordesillas, "Le point culminant de la métrétique », in Christopher C. Rowe (éd.), Reading the Stateman: proceedings of the III Symposium Platonicum, Sankt Augustin, Academia Verlag, 1995; Yvon Lafrance, « Métrétiqe, mathématiques et dialectique en Politique 283 C - 285 c », in Christopher C. Rowe; enfin Gilles Rodier pour lequel la seconde métrétique doit être identifiée à la dialectique supérieure, « Les mathématiques et la dialectique dans le système de Platon », Etudes de Philosophie Grecque, 1924, pp. 37 - 48.

${ }^{47}$ Politique, $285 \mathrm{~d}$.
} 
achevée et définitive du politique, mais qu'il en dresse les principaux aspects. La démarche dialectique mise en œuvre n'est pas totalement aboutie à travers cette détermination de la forme (eidos) du politique: elle était en chemin, sans être parvenu à son point d'arrivée. De ce point de vue, le dialogue éclaire légitimement le politique comme la dialectique, sans les formuler de manière achevée dans les deux cas, en laissant une part d'indétermination caractéristique de l'écriture de Platon. Pourtant, suffisamment d'indices sont laissés à la disposition du lecteur pour que celui-ci puisse envisager de comprendre la triple relation entre politique, métrétique et dialectique. Considérons d'abord l'art de la mesure tel qu'il est décrit dans l'extrait $283 \mathrm{~b}$ - $286 \mathrm{~d}$, dans lequel il n'est pas directement question de la dialectique, mais plutôt de la politique.

Comme nous l'avons vu auparavant, la question de la métrétique est aussi introduite à partir d'une considération sur l'excès et le défaut: "A la longueur, à la brièveté, à tout ce qui est excès ou défaut [...] c'est à tout cela que s'applique l'art de la mesure" ${ }^{2}$. Il s'agit plus particulièrement des contraires concernant la longueur, le grand et le petit, puisque cette idée a surgi après une interrogation sur la juste mesure du discours présent. La discussion sur le politique semble être "trop" longue, alternant échanges dialectiques et récit mythique. L'étranger veut justifier la longueur du discours philosophique à partir d'une mesure ou d'une norme "objective" et non relativement à une comparaison avec ce qui se fait ou ce qui s'est fait. Pourtant, le problème est rapidement élargi à l'ensemble des choses qui relèvent de l'excès et du défaut. L'objet auquel s'applique l'art de la mesure est, comme dans le Protagoras et le Philèbe, clairement constitué. C'est donc sur la compréhension de cet objet que nous allons nous attarder de nouveau. En effet, la nature de l'excès et du défaut est d'être en lui-même indéterminé et inachevé. En eux-mêmes, les contraires sont étrangers à toute mesure et détermination. La possibilité d'être déterminé appartient pourtant à sa nature. Le contraire est en lui-même indéterminable, mais il peut devenir déterminé. Cette possibilité devient effective lorsque la mesure lui est appliquée: être déterminé, achevé revient à recevoir la mesure. En ce sens, l'existence des contraires comme l'excès et le défaut, suppose l'existence de la mesure. Autrement dit, il n'y aurait pas d'excès ni de défaut s'il n'y avait pas

${ }^{48}$ Politique, 283 c $10-283$ d 2. Et 283 c $3-6$. « Examinons d'abord, d'une façon générale, l'excès et le défaut; nous aurons ainsi une règle (kata logon) pour louer ou blâmer à l'occasion ce qu'on aura dit de trop long ou de trop court en des entretiens comme les nôtres ». 
une mesure par rapport à laquelle il serait dit être en excès ou en défaut. Car, d'abord, comment l'excès pourrait-il être sans le défaut, le grand sans le petit? En conséquence de cette relation, la première manière de rendre un contraire déterminable ou mesuré est de rapporter celui-ci à son contraire, déterminer le grand par rapport au petit et inversement. Autrement dit, il s'agit de les rendre commensurables. La production de la commensurabilité, même si c'est une mesure relative, est science et art. Peut-être s'agit-il, au moins en partie, de certains domaines mathématiques qui font intervenir les notions de rapport (logon) et de proportion (summetria). Ces notions interviennent en arithmétique, en géométrie et dans les sciences qui supposent l'application des nombres et des figures en les mesurant les uns par rapport aux autres, comme la musique et l'astronomie. Ainsi, la première métrétique rassemble "tous les arts (sumpasas tekhnas) pour qui le nombre, les longueurs, profondeurs, largeurs, épaisseurs, se mesurent à leurs contraires (pros tounantion metrousin)" "49. Mais, l'excès et le défaut ne supposent pas seulement leur contraire respectif. Car, il faut une juste mesure par rapport à laquelle l'excès puisse être dit en soi excès, et le défaut dit en soi défaut. Par conséquent, la juste mesure est essentielle à tout ce qui est excès et défaut puisque sans elle l'excès ni le défaut ne peuvent être dits ni même produits. L'intérêt d'une telle existence de la juste mesure est de rendre possible de nouveaux arts et de nouvelles sciences: l'art pratique, l'art politique et l'art du discours (rhétorique et dialectique). Le critère de mesure n'est plus l'opposé, mais la "juste mesure (to metrion) elle-même"50. Cette seconde métrétique permet de dépasser la relativité ou la mesure d'une chose par son contraire. Dans les domaines éthiques et politiques, la mesure d'une chose par son contraire, empêche une détermination en soi (ou certains commentateurs disent "absolue") de la réalité, et introduit le risque du renversement et de la contradiction éristiques tels qu'ils peuvent être présentés dans le Philèbe concernant l'un et le multiple. Pour juger de la longueur du discours philosophique, il ne faut pas opérer une comparaison, mais se demander en quel sens ce discours nous approche de la vérité. La mesure relative ou comparative ne peut saisir la valeur du discours: l'excès n'existe que

\footnotetext{
49284 e $3-5$.

50 « Pour diviser la métrétique de la façon que nous disions, nous n 'aurions qu'à y distinguer les sections suivantes: nous mettrions, d'une part, tous les arts pour qui le nombre, les longueurs, profondeurs, largeurs, épaisseurs, se mesurent à leurs contraires, et, de l'autre, tous ceux qui se réfèrent à la juste mesure ».
} 
relativement à un autre discours ce qui n'est pas un critère suffisant. De ce point de vue, il est nécessaire de s'élever à une mesure "en soi” et non "relative à" (pros allêla). C'est précisément ce qui différencie les deux sortes de métrétique présentées dans le dialogue: "nous voilà donc contraints d'admettre, pour le grand et le petit, deux modes d'existence et deux jauges: il ne faut point, en effet, nous en tenir, comme nous le faisions tout à l'heure, à leur rapport mutuel, mais plutôt distinguer, comme nous le faisons maintenant, d'une part le rapport qu'ils ont l'une à l'autre, et, d'autre part, celui qu'ils ont à la juste mesure" 51 . La mesure "en soi" prend donc le nom de "juste mesure" (metrion). Nous pourrions plutôt caractériser cette distinction en différenciant les objets auxquels chaque partie de la métrétique s'applique: la seconde métrétique rassemble sous son chef les actions, les discours, les actes et de nombreux arts, dont l'art politique. L'ensemble de ces objets sont susceptibles d'accepter la "juste mesure"; autrement dit, l'essence de leur vertu réside dans leur juste mesure.

La seconde métrétique ne se limite pas à l'art du discours. En effet, la juste mesure est présentée comme la condition de possibilité de tout art: "la mesure (metrêseôs) en effet, tous les oeuvres d'art y participent en quelque façon" ${ }^{2}$. En ce sens, il existe une relation intrinsèque entre l'art et la juste mesure (metrios): "tous les arts sont (tas technas pasas einat)" parce qu'il y a "production de la juste mesure (tên tou metriou genesin)" 53 . Or, la juste mesure est produite par l'application du metron: "on peut dire que, pour tous ces arts, ce qui serait en-deçà ou au-delà de la juste mesure n'est point une chose irréelle; c'est, au contraire, une réalité fâcheuse, qu'ils veillent à écarter de leurs productions (tas praxeis) et c'est en préservant ainsi la mesure (to metron sôizousai) qu'ils assurent la bonté et la beauté de leurs oeuvres (panta agatha kai kala apergazontai) ${ }^{54}$.”. Le metrios est ce qui est produit dans l'objet, alors que le metron est ce qui est préservé ou respecté dans la production: le metron est le principe sur lequel l'agent se fixe pour produire la juste mesure. Les techniques existent, et la raison de cette existence est la présence dans les oeuvres produites de la juste mesure ou du mesuré. Nous supposons donc que la seconde métrétique a pour objet des réalités prises dans la génération, le devenir et même le sensible, et qu'elle ne désigne pas

51283 e $8-12$.

52285 a 3.

53284 d $4-6$.

${ }^{54} 284$ a $8-284$ b 1. 
directement une métrétique intelligible ou la dialectique comme si elle était séparée du devenir. Platon insiste suffisamment sur l'objet de la seconde métrétique pour que cela soit presque sans doute. Ainsi, "ce mot que beaucoup d'hommes d'esprit se plaisent à répéter comme une profonde maxime, que la science de la mesure (metrêtikê) s'applique à tout ce qui devient (peri pant' esti ta gignomena) nous sommes précisément en train de le redire" 55 . Ensuite, l'objet de la seconde métrétique est déterminé par Platon: il s'agit de la longueur, du grand et du petit d'abord dans les discours, puis il y a un glissement vers l'excès et le défaut concernant les praxeis, les erga et les technai. Or il est clair que la praxis, l'ergon et la technê se trouvent dans le devenir (284 a - c). "N'allons-nous pas anéantir l'art politique, etc? [...] Car, si l'on ne nous concède pas cela, il n'est pas possible d'établir qu'il puisse y avoir un politique, ni quelque autre type d'homme possédant un savoir qui s'applique à l'action (peri tas praxeis epistêmona)". Ces termes erga, apergazomai, praxis et le lexique lié à la genesis confortent l'hypothèse selon laquelle cette métrétique est pratique. Une autre caractéristique de la description faite par Platon au sujet de la seconde métrétique offre à notre hypothèse un nouvel argument. En effet, à diverses reprises, Platon déclare que le metrios, la juste mesure, advient à la génération en 284 c 1, $284 \mathrm{~d} 6$ (tên tou metriou genesin). Elle est produite dans le devenir. Que la métrétique soit une technê, cela signifie finalement qu'elle est une application au devenir et au sensible: elle est fondamentalement pratique au sens où son objet est l'action en général. La métrétique qui participe à l'art politique se "réfère [donc] à la juste mesure, à ce qui est convenable, opportun, requis, à tout ce qui tient le milieu entre les extrêmes (pros to metrion kai to prepon kai ton kairon kai to deon kai panth ' hoposa eis to meson apôikisthê tôn eskhaton)". Les différents termes utilisés désignent sans aucun doute des qualités propres à l'action humaine en général à la fois dans sa dimension morale, éthique et politique. Le to prepon exprime l'idée d'une convenance en soi ou absolue qui ne se mesure plus à l'aune des contraires, mais qui indique la conformité à une norme intelligible et séparée qui vient donner aux réalités en devenir leur essence et leur forme. Sans cette application de la norme intelligible ou de la forme, la réalité en devenir reste nécessairement imparfaite et inachevé.

55285 a $1-2$. 
Malgré le caractère inachevé de la définition du politique par Platon, il est donc possible de formuler l'essence du politique, sans considérer cette formule comme valant absolument et définitivement pour le philosophe. Nous relèverons trois traits principaux de l'art politique, selon sa nature scientifique, sa finalité et son objet pratique: il s'agit, d'abord, d'une véritable science de type "directive"; ensuite, la finalité de la science politique consiste à déterminer ce qui est convenable pour la cité en vue de l'améliorer et de l'éduquer à la vertu; enfin, l'homme politique est législateur et "tisserand", c'est-à-dire qu'il "tisse" les liens sociaux entre les citoyens ou les éléments de la cité. Le Politique est principalement un homme qui possède la "science" et "l'art". Cette science est supérieure par son objet et par le caractère exceptionnel de celui qui la possède. L'art politique consiste "à vouloir et pouvoir gouverner avec vertu et science" 56 en suivant cette règle selon laquelle il faut "distribuer en toute occasion entre les citoyens une justice parfaite, pénétrée d'esprit et d'art, et réussir ainsi à les préserver (sôzein), mais encore, autant que possible, à les rendre meilleurs" ${ }^{\text {"57. }}$. Cette science n'est pas entièrement théorique, mais elle a pour finalité de commander l'action et la production dans la cité: la division initiale des "sciences théoriques (gnostikê)" en "critique" et "directive" est certainement encore valable à la fin du texte ${ }^{58}$. La fonction essentielle de l'art politique, son ergon propre, est de commander la cité et les citoyens; son but est de les préserver et de les rendre meilleurs par ce commandement. Le Roi est celui qui est "archontas"59, qui dirige la cité, qui en possède et fournit l'archè: l'art politique est "la science du gouvernement des hommes" 60 . La possession de la science est le principe premier de toute classification et distinction entre les hommes politiques, les cités et les constitutions. C'est la présence de la science ou son absence qui différencie les constitutions ${ }^{61}$. Les autres principes, obéissance à la loi, liberté des citoyens, sont toujours considérés secondaires. Or seul un nombre restreint d'individus peut réaliser cette science, d'où la préférence à la monarchie et à l'aristocratie par différence à la démocratie du point de vue de la cité idéale ${ }^{62}$. La science politique ne peut pas raisonnablement être partagé par le grand nombre. Platon répond à

\footnotetext{
$56301 \mathrm{~d} 1-2$.

57297 a 7 - b 3.

58260 b; 292 b.

59290 b $5-6$.

60292 d $3-4$. Ainsi, l'art politique gouverne les arts éducatifs qui sont spécialisés dans telle ou telle tâche. cf. $308 \mathrm{~d}$ e.

$61293 \mathrm{c}-\mathrm{d}$.

62292 e - 293 a; 296 d - 297 c.
} 
la thèse de Protagoras développée dans le dialogue éponyme selon laquelle l'art politique serait partagé entre tous. Mais ce que Platon critique plus encore, ce sont les "imitateurs déviants" du savoir véritable, les sophistes. Une opposition stricte est faite entre celui qui possède un "savoir véritable"63 et celui qui ne possède qu'une apparence de savoir ou la simple opinion. Cette opposition est réalisée la première fois dans le cadre de la dénonciation des rivaux du politique. Il faut séparer du politique véritable "le choeur qui s'agite autour des affaires politiques" c'est-à-dire "le plus magicien des sophistes, le plus consommé dans cet art, celui qu'il est si difficile de distinguer d'avec les vrais politiques et les vrais hommes royaux, mais que nous en devons pourtant distinguer, si nous voulons découvrir clairement l'objet que nous cherchons. ${ }^{64 “}$ Ce qui caractérise l'art du sophiste, c'est l'imitation décadente et pernicieuse, imitation d'un savoir véritable mais qui n'est en réalité qu'apparence, et qui, de plus, est une corruption de l'état des cités ${ }^{65}$.

Dans cette perspective, la pratique consiste effectivement dans la droite détermination de ce qui est le meilleur, pour ainsi dire à chaque instant de la cité. L'art politique réside dans la capacité à commander la réalisation de l'action droite et vertueuse dans le monde humain en devenir grâce à une science supérieure des Idées. Par cette science supérieure, le Roi (ho Basileus) sait ce qu'il faut faire et à quel moment le faire dans le temps propre à la cité. Il s'agit maintenant de la notion de kairos. Si ce terme peut être envisagé dans la pensée archaïque dans sa dimension générale de convenance, il est sans doute employé par Platon à la suite des sophistes dans un sens temporel. L'art politique s'inscrit essentiellement dans le temps humain, il ne peut le nier. Le Roi doit toujours évaluer le chemin qui sépare la cité réelle de la cité idéale et prendre des décisions en fonction de l'éloignement et de l'orientation prise par la cité. Il peut selon le moment transformer les lois et même les transgresser quand cela est utile: "le véritable politique, s'inspirera, dans bien des cas, uniquement de son art et, pour sa propre pratique, ne se souciera aucunement

\footnotetext{
63292 b 293 c

${ }^{64} 291$ c 1 - 6. En 303 b 8 - 303 c 5, Platon déclare avec force que « tous ceux qui ont un rôle dans ces constitutions, celle qui est scientifique exceptée, sont à rejeter comme n'étant pas des politiques, mais des factieux, meneurs de pires illusions, illusions eux-mêmes, mimes et charlatans insignes et, à ce titre, sophistes insignes entre tous les sophistes $»$.

$65293 \mathrm{c}$ - d. «Parmi les constitutions celle-là sera éminemment et uniquement la droite constitution, qui montrera des chefs doués d'une science véritable et non d'un semblant de science, et, que ses chefs s'appuient sur des lois ou s'en passent, qu'ils soient agréés ou subis, riches ou pauvres, rien de tout cela ne doit compter dans l'appréciation de cette norme droite. »
} 
de la lettre écrite, s'il trouve qu'une façon nouvelle d'agir vaut mieux que les prescriptions rédigées par lui et promulguées pour le temps de son absence" 66 . L'art du politique est à la fois une connaissance de la norme ou de la vérité du Bien politique, et une capacité à la réaliser en pratique, de faire advenir la norme dans le devenir et le sensible en reconnaissant les moments opportuns à cette application. Or, comme l'action humaine se déroule entre les contraires, l'acte politique sera toujours une élévation hors de la contrariété pour saisir la forme convenable à donner aux actes et aux lois, forme qui se confondra dans biens des cas avec le "juste milieu" qui assure aux actions leur participation au beau et au bien.

$\mathrm{Si}$, idéalement, le mode d'action de l'homme politique serait d'intervenir à chaque instant et auprès de chaque particulier en vue de dicter l'action droite, dans la pratique humaine, la législation est un acte fondamental de l'exercice politique. La tension entre le caractère défectueux des lois et leur nécessité est à l'origine du rapport ambigu que l'homme politique entretient avec les lois. Le véritable politique peut enfreindre les lois car son art lui permet d'améliorer la cité, alors que les autres "législateurs", et surtout les sophistes, corrompent la cité en enfreignant les lois ( $300 \mathrm{~b}$; $300 \mathrm{~d}-\mathrm{e})$. La science est supérieure aux lois $^{67}$, alors que l'ignorance est inférieure aux lois. L'homme politique peut enfreindre les lois, car "il pose sa propre science comme loi" ${ }^{68}$. Si d'un côté, il peut s'en affranchir, d'un autre côté la loi est un instrument nécessaire du commandement politique. L'idéal serait que l'homme politique puisse juger et commander tous les cas particuliers $^{69}$, or c'est impossible. Il a donc recours à la loi comme un instrument général qu'il doit savoir adapter aux situations et aux changements de la société comme le capitaine s'adapte à la situation maritime ou le médecin qui adapte son traitement par rapport à l'évolution de la maladie de son patient. Par conséquent, l'art politique devient nécessairement, malgré les défauts de la loi, l'art d'écrire des lois justes suivant la norme de la droite constitution: l'objet du politique est le juste et l'injuste, et son acte est la formulation droite des lois qui déclarent ce qui est juste et ce qui est injuste. Le politique est donc législateur. Il est seul véritable législateur puisqu'il est le seul à pouvoir légitimement transformer les lois en vue d'améliorer la cité par la

\footnotetext{
66300 c $7-300$ d 2.

67297 a 7.

68297 a 5.

69295 a 10 - 295 b 1. «Comment serait-on jamais capable de venir s'asseoir, à tout instant de la vie, auprès de chaque particulier pour lui prescrire exactement ce qu'il doit faire? »
} 
science $^{70}$ et à déterminer ce qui convient (to prosêkon) $)^{71}$ au moins dans la plupart des cas, si ce n'est en légiférant sur l'ensemble des cas. Ce qui fait la légitimité de l'action politique, c'est son fondement scientifique et philosophique.

La seconde manière de comprendre la pratique politique est la référence faite au paradigme du tissage à partir de $279 \mathrm{a}$. Nous pouvons considérer que l'art politique consiste en un entrelacement et un mélange des éléments qui constituent un tout. Il s'agirait ainsi de tisser les liens entre des éléments épars, disparates et même contradictoires en vue de former une totalité unifiée. La cité est en ce sens comparable à un cosmos, ou au corps humain. La même analyse s'applique à ces différentes réalités: il est nécessaire de produire l'harmonie entre les éléments en introduisant, comme le montrent le Philèbe $e^{72}$ ou encore le Timée, la mesure dans l'ensemble. Autrement dit, il est nécessaire d'opérer le bon mélange entre les éléments opposés qui constituent un tout ordonné (kosmos). Selon le paradigme du tissage, l'art politique (comme peut-être la dialectique) pourrait être considéré comme la capacité à "faire un entrelacement" (sumplokê). La première opération dans l'art du tissage est d'entremêler les différents fils, et par cette opération, produire une oeuvre unie rassemblant ce qui, à l'origine, était dispersé. Nous pensons voir une illustration de ce processus appliqué à l'art politique dans le dernier moment du dialogue: "ne voudrons-nous pas utiliser notre paradigme du tissage pour expliquer la politique"73. Il s'agit donc "exposer la royale fonction d'entrecroisement (Tên basilikê sumplokên), sa nature et sa manière d'entrecroiser"74. Pour couvrir cet objectif, il faut d'abord montrer que l'objet politique accepte les contraires. Si l'objet de la politique est d'éduquer les citoyens à la vertu, il faut, alors, reconnaitre une forme de contrariété entre les différentes parties de la vertu. Ce propos apparaît d'autant plus surprenant dans la bouche de Socrate après l'analyse du Protagoras dans lequel celui-ci semblait plutôt montrer l'unité et l'amitié entre les parties de la vertu. Cependant, comme nous le verrons concernant la dialectique, l'unité de la vertu est faite d'une multiplicité qui les unes à l'égard des autres peuvent se différencier par des propriétés spécifiques les séparant. Cette différenciation est aussi constituée

70 De multiples passages expriment cette idée parmi les quels 295 e - 296 a.

71295 a 2.

72 Un passage du Philèbe est à ce sujet significatif en $25 d-26 d$, dans lequel santé, musique, saisons et pour ainsi dire l'ensemble de l'univers organisé est le résultat de l'introduction de la mesure dans des éléments à l'origine éparses et indéfinis.

73305 e $8-10$.

74306 a $1-3$. 
d'après le critère du moment opportun (kairos) puisque la qualité propre d'une vertu peut dans telle ou telle circonstance ne peut pas être appropriée. L'étranger illustre son propos en prenant l'exemple du courage (andreia) et de la sagesse (sôphrosunê) à partir de 306 a en montrant que les qualités propres au courage (promptitude, vitesse, énergie) s'opposent aux qualités propres à la sagesse (lenteur, douceur, caractère paisible). Or, c'est selon les circonstances que l'une ou l'autre vertu est requise en vue de produire l'acte vertueux. En ce sens, Platon renoue avec la tradition archä̈que sur ce qu'est l'aretê. l'excellence, la capacité à agir de manière appropriée dans une situation donnée. Il en est ainsi des vertus dans leur dimension pratique. Leurs fondements philosophiques et épistémiques ne remettent pas en cause le fait qu'elles doivent s'appliquer dans le devenir et ainsi obéir à des critères "pratiques" tels que le moment opportun, le convenable et le juste milieu ${ }^{75}$. Nous comprenons une nouvelle fois la définition de la seconde métrétique. La politique est une métrétique dans la mesure où elle doit opérer l'entrelacement ou le mélange approprié entre des qualités contraires, et puisqu'elle est la détermination de ce qui est mesuré. En effet, l'entrelacement doit aboutir à un mélange mesuré entre les extrêmes. Les deux vertus et leurs propriétés sont identifiées à des caractères humains ou à des types d'hommes ${ }^{76}$. Autrement dit, la cité est composée de types d'hommes caractérisés par des propriétés et des vertus différentes et même opposées quant à leur mode de réalisation. Et à l'intérieur même de chacun, il existe deux opérations d'entrelacement, l'une divine (to theion) et l'autre humaine (anthrôpinos). Nous sommes composés d'une partie divine et d'une partie humaine et animale. Il faudra lier la première à la vérité, à l'opinion droite et à la connaissance, et de cette manière assurer la vertu en chacun ${ }^{77}$. Quant à la seconde, c'est en assurant les échanges entre les types opposés d'hommes tant au point de vue politique que de celui du mariage qu'il est possible d'opérer le "mélange" harmonieux ${ }^{78}$ : "c'est là toute la fonction de ce royal art de tissage, de ne jamais laisser le divorce s'établir entre le caractère tempéré et le caractère énergique, de les ourdir ensemble"79.

\footnotetext{
75 Deux expressions construites sur le terme kairos sont utilisées, en kairôi (307 b 1), akaira (306 b 6), tou kairou (310 e 2).

${ }^{76} 307$ e - 308 b. « N'avons-nous pas vérifié le premier point de notre enquête, à savoir que certaines parties de la vertu, et non des moindres, sont opposées entre elles par nature, et engendrent, dans les esprits où elles résident, les mêmes oppositions? »

77309 e -310 a.

$78310 \mathrm{a}-311 \mathrm{c}$.

79310 e $7-9$.
} 
A ce point de notre étude, un principe est acquis: la vertu et l'art politique reposent sur la science; et cette science peut être caractérisée comme un art de la mesure. Cependant, il nous faut encore approfondir la compréhension de l'aspect épistémique de la vertu et de l'art politique en se demandant quelle peut être la fonction de la dialectique dans la constitution des arts pratiques. Corrélativement, l'art de la mesure tisse des relations précises avec l'art dialectique, et il est dans notre devoir de comprendre la nature de ces liens.

\section{Le fondement dialectique de l'art de la mesure en éthique et en politique.}

Au cours de notre investigation à l'intérieur du Protagoras et du Politique, nous avons constaté la relation intrinsèque entretenue selon Platon entre technê et epistèmè, entre praxis et epistèmè. L'art d'agir est fondamentalement lié à une connaissance. Plus précisément encore, il n'y a pas de vertu sans une science qui en soit au fondement. La science est donc au fondement de la vertu et de l'art. Dans le Protagoras, cette idée est développée concernant le mode de vie éthique; dans le Politique, l'action et l'art politiques sont clairement et avec insistance associés à la science. Nous nous interrogerons donc sur ce qu'est cette science non plus dans son aspect pratique et directif, mais dans son aspect gnoséologique et ontologique. Le problème principal que nous voulons désormais formuler porte sur la relation entre cette science revendiquée au fondement de l'éthique et de la politique et la dialectique. La dialectique possède-t-elle une fonction dans la constitution de ces sciences. Corrélativement, quel(s) lien(s) pouvons-nous établir entre métrétique et dialectique de ce point de vue? Par exemple, si nous nous référons au Politique, dans quelle mesure métrétique, dialectique et politique sont apparentées ou liées? Et de quelle manière désigner cette relation? Est-ce une relation de communauté comparative dans la méthode, au sens où elles emploieraient une méthode comparable? Ou faut-il plutôt concevoir une identification de ces différents arts? Ou encore faut-il considérer la dialectique comme la science première qui guiderait en quelque sorte les autres dans leurs applications?

Le Protagoras fait à ce sujet mention d'une mystérieuse science. Socrate indique l'existence de celle-ci comme si elle était au principe de la métrétique: "Quelle science et quel art, nous le verrons plus tard. Mais que ce 
soit une science, cela me suffit pour la démonstration" 80 . Platon veut-il indiquer par cette allusion l'art dialectique puisqu'il ne désigne pas la métrétique? C'est, dans tous les cas, une hypothèse qu'il faut prendre au sérieux. Cette recherche ultérieure fait-elle référence à une enquête réalisée dans un autre dialogue? Fait-elle référence au problème concernant la relation entre métrétique et dialectique tel qu'il est présenté au moins de manière implicite dans plusieurs dialogues tels que la République, le Philèbe et le Politique? De même dans le Politique, après avoir opéré la division de la métrétique en deux, Socrate évoque alors l'idée selon laquelle l'exercice dialectique serait l'objet réel du dialogue ${ }^{81}$. La définition du politique ne serait qu'un "jeu” pour exercer le jeune Socrate à la dialectique. Nous ne croyons pas absolument à cela. En revanche, deux idées peuvent être retenues par cette déclaration. La première déjà indiquée rappelle que la définition du politique n'est pas reconnue par Platon comme définitive, totale et achevée. La seconde qui nous importe désormais est la place donnée en filigranes à l'art dialectique dans le dialogue. Platon semble utiliser une succession de paradigmes qui s'éclairent linéairement les uns après les autres pour s'achever dans une indication "paradigmatique" de la dialectique par la métrétique et la politique. En effet, l'art du tissage éclaire l'art politique qui est ramené à l'art de la mesure. Ce dernier illustre une méthode ou la méthode de division et de synthèse utilisée par l'art dialectique. Ainsi, deux opérations essentielles constituent l'ensemble de ces arts à des niveaux de réalités différents: le tissage est rassemblement et "démêler ce qui était uni et pressé ensemble (To tôn sunestôtôn kai sumpepilemenôn)"82. Or, le politique est constitué sur le paradigme du tissage comme nous l'avons vu (en particulier pour le rassemblement). Quant à la dialectique, elle est clairement une méthode consistant à diviser et rassembler. L'opération principale utilisée dans le Politique est la division, c'est-à-dire "démêler ce qui était uni et pressé ensemble" dans un seul même nom ou dans un seul et même genre en distinguant les espèces et les parties appartenant à ce genre mais se différenciant par quelque propriété spécifique. Pourtant, dans ce dernier dialogue, la dialectique n'est pas clairement illustrée par la métrétique. C'est

\footnotetext{
80357 b $4-5$.

81 En Politique 285 d, L'Etranger déclare: « Que dirons de notre enquête au sujet du politique? Est-ce directement par intérêt pour lui que nous nous la sommes imposée, ou bien est-ce pour devenir meilleurs dialecticiens sur tous les sujets possibles? »

82281 a $5-6$.
} 
dans le Philèbe où ce rapprochement est effectué de manière plus évidente. Ainsi, dans ce dernier dialogue, la méthode consistant à rassembler le multiple dans l'un et diviser l'un en sa multiplicité sont présentées comme les deux opérations essentielles de la dialectique. La méthode dialectique est alors définie comme un art de la mesure. L'art de la mesure intervient donc dans différents arts et sciences, des arts pratiques à la science dialectique. Cependant, une question reste ouverte concernant la relation entre métrétique et dialectique. Est-il possible d'effectuer une identification entre les deux? Dans tous les cas, cela nous apprend que l'art de la mesure peut s'appliquer à différents objets et à différents niveaux de réalité. Le problème principal que nous voulons examiner consiste à se demander si la dialectique peut être considérée comme le fondement épistémologique de l'éthique et de la politique selon Platon. Cette question peut être étudiée par le biais de la métrétique en s'interrogeant sur la possibilité que celle-ci soit un art s'appliquant à l'ensemble des sciences considérées.

La production de la juste mesure dans le devenir n'est pas effectuée au hasard, de manière relative ou encore proprement humaine. En effet, cette production est en étroite relation avec l'ousia des choses prises dans le devenir comme le souligne une indication du Politique. Dès lors, il faut reconnaître que la mesure a deux registres d'application, un registre gnoséologique et un registre ontologique. Suivant la perspective gnoséologique c'est la mesure qui rend connaissable; d'un point de vue ontologique, c'est la mesure qui rend achevé et parfait (telos). Or, il semble que la mesure gnoséologique, du moins en ce qui concerne la dialectique de l'un et du multiple (diérèse de l'un et synérèse du multiple), doit se conformer aux mesures naturelles ou plutôt essentielles des choses. La mesure en soi est-ce déterminer ou produire une réalité en devenir selon une forme intelligible? Nous serions tentés par l'interprétation selon laquelle la mesure en soi doit être identifiée à une forme, eidos ${ }^{83}$. Si la mesure en soi de la seconde métrétique du Politique est telle qu'elle est la réalisation de la forme

\footnotetext{
${ }^{83}$ Cornelius Castoriadis, Sur le Politique de Platon, Paris, Editions du Seuil, 1999, p. 110 - 111. « Toutes ces mesures (de la première métrétique) sont relatives, c'est évident, mais si toute mesure relative présuppose une mesure qui n'est pas relative, vous arrivez nécessairement à la nécessité pour penser, pour parler, vous arrivez à l'idée qu'il y a quelque chose qui est mesure du reste, pas relativement, mais qui est mesure absolue, qui est norme, qui est donc une Forme, un eidos. [...] II y a donc une dimension de mesure relative mais elle n'est là que pour instrumenter l'incarnation d'une forme qui, elle, n'est pas relative à autre chose, qui n'est relative qu'à la beauté, à la forme. C'est cela que Platon appelle metrion. »
} 
dans le devenir, alors elle fait de la dialectique le fondement de la politique en ce que la dialectique est la seule science qui est finalement la compétence requise pour déterminer l'essence des choses. Le Philèbe et le Timée confirment au sein du registre cosmologique cette idée. Lors de la génération $\mathrm{du}$ monde, les mesures et les proportions sont appliquées aux réalités en devenir et sensibles qui sont par nature imparfaites et inachevées. C'est l'application de la mesure qui les fait advenir dans un ordre déterminé et achevé de telle sorte qu'elles s'insèrent dans une harmonie et donnent lieu à des réalités belles et bonnes. En ce sens, l'homme, d'un point de vue politique, est comparable, jusqu'à un certain point au démiurge, en ce qu'il doit appliquer la mesure aux réalités multiples et changeantes pour leur faire adopter la forme convenable. Cette application suppose une connaissance de ce qui fait la véritable valeur des choses. C'est pourquoi nous pouvons en déduire que cette connaissance relève d'une science intelligible dont l'objet sont les essences (ousiai) et les formes (idea dans le Philèbe). L'analogie avec le démiurge divin est opérante pour comprendre l'action de l'homme sur le devenir. Le politique est celui qui produit le monde humain suivant la norme intelligible. La juste mesure correspond à la réalisation dans le monde humain de l'essence des choses connue par l'homme politique et dialecticien qui emploie cette connaissance comme mesure pour réaliser l'essence intelligible dans la cité. La nécessité du devenir indique la nature de la génération qui risque toujours l'excès et le défaut, autrement dit l'imperfection et l'inachèvement; mais en même temps, sa nature est de recevoir une mesure et une forme déterminées. Le devenir et le sensible se caractérisent par cette potentialité. Dans la pratique politique, cela signifie que le Roi ne peut réaliser le bon mélange, l'entrelacement correct sans connaitre l'essence des choses entrelacées et leurs propriétés spécifiques intelligibles. De même, la démarche méthodologique de la dialectique dans la détermination des formes spécifiques aux Idées se constitue sur les deux opérations de la division et de la synthèse. Ces deux opérations qui définissent en grande partie la dialectique font appel à l'idée de mesure et à l'activité de mesurer. En effet, le rassemblement du multiple dans l'un est un acte de mesure ${ }^{84}$, et la division de l'un en ses différentes parties doit se faire

${ }^{84}$ Philèbe, 16 d-e. « II nous faut donc, puisque les choses sont ainsi ordonnées (diakekosmêmenôn), poser toujours, en quelque ensemble que ce soit, et chercher en chaque cas une forme unique (mian idean) - on l'y trouvera en effet présente [...]. Quant à la forme de l'illimité (tên de tou apeirou idean), ne 
selon des mesures et des nombres précis ${ }^{85}$, conformes à la nature des liens essentiels entretenus antre les différentes espèces qui appartiennent à un genre $^{86}$. Dans cette perspective, la détermination des intermédiaires (mesa) et des mesures (metra) qui forment les relations entre l'un et le multiple est le fondement de la dialectique et l'opération essentielle qui la sépare de l'éristique ${ }^{87}$. L'art de la dialectique est alors illustré par différents paradigmes, l'art musical et l'art grammatical. Ces deux arts portent sur des objets, le son et la voix, qui s'insèrent dans le cadre de l'un et du multiple. D'une part, ils présentent une forme unique, et d'autre part, ils présentent une infinité de possibilités. L'art consiste à déterminer l'unité puis à saisir les mesures et les intermédiaires qui mènent à la multiplicité en divisant correctement, selon la nature des choses, le genre unique. En musique, il faut saisir le nombre d'intervalles qui nous fait passer de l'unité du son à la multiplicité des sons, "rapports qui se mesurent par des nombres et qu'il faut, disent les anciens, appeler rythmes et mètres (di' arithmôn metrêthenta dein au phasi puthmous kai metra eponomazein)" 88 . Le terme metra semble s'appliquer ici à un nombre restreint de sciences, comme la musique et l'astronomie. Cependant, au regard de l'emploi général du terme fait dans le cadre de la description du genre du limitant, il apparaît que cette notion possède une valeur d'ensemble pouvant s'appliquer à toutes les opérations consistant à déterminer la valeur d'une chose que ce soit quantitativement ou qualitativement. La connaissance des essences et de leurs relations est le principe des arts tels que la politique. D'une certaine manière, la dialectique peut être comprise comme une métrétique intelligible dont l'objet est l'ensemble des essences, des formes et de leurs relations d'appartenance ou d'opposition. Les arts sont l'application dans le devenir et le sensible de la connaissance dialectique dans un secteur délimité. Leur finalité est véritablement de réaliser "matériellement" les

pas l'appliquer à la multitude avant d'avoir saisi quel nombre total celle-ci réalise dans l'intervalle entre l'un et l'illimité (ton arithmon autou panta katidê ton metaxu tou apeirou ten kai tou henos). »

$8516 \mathrm{~d}$. « Si donc nous appréhendons [cette unité], après cette unique forme, il faut examiner s'il y en a deux, et, sinon deux seulement, trois ou quelque autre nombre (arithmon); puis faire le même examen pour chacune de ces unités nouvelles, jusqu'à ce que, de cet un primitif (to kat' arkhas hen), on voie non seulement qu'il est un et multiple et infini (hen kai polla kai apeira), mais aussi quelle quantité (hoposa) précise il atteint. »

${ }^{86}$ Philèbe, $12 \mathrm{e}-18 \mathrm{~d}$.

8717 a. "Quant aux intermédiaires, ils les ignorent (ta de mesa aitous ekpheugei), alors que les respecter est ce qui distingue, en nos discussions, la manière dialectique de la manière éristique (to te dialektikôs pasin kai to epistikôs hemas poieisthai pros allêlous tous logous) ».

$8817 \mathrm{~d} 5-6$. 
relations intelligibles en faisant advenir cet intelligible dans le devenir et le "corps" même des choses. Autrement dit, c'est donner naissance à la juste mesure. Nous retrouvons là la notion de mélange. Il s'agit non seulement d'un mélange entre éléments physiques, mais surtout d'un mélange entre deux niveaux de réalités, l'intelligible advenant dans le devenir, ou selon les termes du Politique le divin advenant dans l'humain et le vivant. Ainsi, dans le Philèbe a lieu "cette découverte, que c'est la mixis, qui produit la genesis '89. Ce problème de la mixtion (meixin kai krasin, 64 a du Philèbe) est un problème qui s'étend de l'homme au Tout (en t'anthrôpôi kai tôi panti $)^{90}$. L'Idée de Bien peut même être découverte et appréhendée par l'intermédiaire des réalités mixtes les plus parfaites: "le plus beau mélange, le composé le mieux assuré contre tout discord (kallistên idonta kai atasiastotatên meixin kai krasin)" est celui dans lequel nous pouvons "s'efforcer d'y découvrir (mathein peirasthai) quel est le bien dans l'homme et dans le Tout et quelle nature nous devons lui supposer (en teutêi mathein peirastai ti pote en t' anthrôpôi kai tôi panti pephuken agathon)" "11. Or, ces êtres sont nécessairement mélangés à la Vérité, comme si la Vérité était garante de la perfection de l'ensemble. Mieux encore, il n'y aucune génération et existence sans intervention de la Vérité. A ce titre, le cosmos n'est pas pure matière, pur devenir, mais il est le produit de la Vérité: "ce à quoi nous ne mêlerons pas la vérité, cela ne pourra jamais vraiment naître ni, une fois né, vraiment exister" 92 . Or, la Vérité est fondamentalement d'ordre intelligible, ce qui signifie que le mélange réalisé mêle l'intelligible au devenir sensible. Par cet entrelacement et composition, nous nous rendons alors "aux grandes entrées du Bien” (64 c 2). Ce passage éclaire l'expression du Politique selon laquelle la seconde métrétique s'applique "à l'essence nécessaire du devenir (kata tên tês geneseôs anankaian ousian)" ${ }^{\prime 3}$. Le devenir dans son aspect cosmique est soumis à une nécessité qui est celle de l'essence intelligible des Idées. Autrement dit, le monde est produit suivant un modèle divin ou la norme intelligible du Bien.

\footnotetext{
89 Pierre Maxime Schuhl, « Sur le Mélange dans le Philèbe », Revue des Etudes Grecques, 80, 1967, pp. 220 - 226, p. 222; voir aussi du même auteur, « Genesis et répétition », Revue Philosophique, 1967, I, pp. $120-121$.

9064 a $1-2$.

9163 e $8-64$ a 4.

9264 b $2-3$.

93283 d $8-9$.
} 
Pour conclure, cette description qui s'applique à l'homme et au tout montre que la vie humaine est caractérisée par ce mélange et cet entrelacement. Autrement dit, l'éthique est la capacité d'harmoniser sa vie humaine faite de plaisirs et de douleurs avec la Vérité et le Bien, en faisant que ce soit la partie divine et intelligible de notre âme qui guide et commande notre nature imparfaite. Bien entendu, le même raisonnement est consacré à l'organisation de la cité. Ethique et politique reposent donc sur la dialectique puisque cette dernière est la connaissance de la norme intelligible permettant aux arts humains de se réaliser; et en tant qu'arts, leur finalité est de produire la vertu dans leurs oeuvres. La métrétique intervient dans une double dimension: premièrement, la méthode dialectique emploie une méthode métrétique; deuxièmement, les arts dont l'action fondamentale est d'opérer le mélange et l'entrelacement entre intelligible et sensible relèvent de cet art de la mesure ${ }^{94}$.

E-mail: sebastien.bassu@gmail.com

Recebido: julho/2009

Aprovado: agosto/2009

${ }^{94}$ Nous indiquons que cet article participe à l'élaboration de notre thèse sur la notion de «mesure »: «le metron entre logos et praxis dans la philosophie grecque». Une vue d'ensemble de la notion de mesure chez Platon est aussi présentée et organisée dans le cadre d'une conférence à publier donnée à Aix-en-Provence le 08 avril 2009, grâce à l'Institut d'Histoire de la philosophie, dirigé par M. Alonso Tordesillas, sous le nom de «Platon, la mesure et ses mesures ». 